\title{
Chemical and Physical Properties of Adulterated Honey and Developing Means of Identifying Adulterants
}

\author{
Meseret Gemeda*, Deresa Kebeba, Teferi Damto, Gemechis Legesse \\ Holeta Bee Research Center, Oromia Agricultural Research Institute, Ethiopia
}

*Corresponding Author: Meseret Gemeda, Holeta Bee Research Center, Oromia Agricultural Research Institute, Ethiopia

\begin{abstract}
Honey is defined as a naturally sweet mixture produced by bees (Apis mellifera) from the nectar or from secretions of living parts of plants or excretions of plant sucking insects on the living parts of plants transform and combine with specific substances of their own (such as enzymes), deposit, dehydrate, store and leave in the beeswax honeycombs to ripen and mature. It is typical sugary foodstuff; according to present-day regulations, apart from other forms of honey no other or additives can be added to it. The aim of this study was to develop identification means of adulterated honey using chemical and physical analysis. Adulteration of honey was obtained by mixing and homogenizing major honey adulterants so far known like sugar, banana, "sheb", molasses and water with different ratio. Moisture content, electrical conductivity, PH, ash, free acidity (FA) and Hydroxymehylfurfural of adulterated and pure honey were determined. The pure honey free acidity content is significantly different from adulterated honey in all treatments group $(P<0.05)$. Highest FA content $(458.33 \pm 1.66 \mathrm{meq} / \mathrm{kg}$ ) recorded in (honey: sugar: water: sheb) treatment. Adulteration affects the purity and quality properties of honey, by using the ash content, free acidity and electrical conductivity adulteration can be identified and color parameter is not recommended for identification of adulterated honey.
\end{abstract}

Keywords: adulteration, adulterant, honey, chemical properties

\section{INTRODUCTION}

Honey is defined as a naturally sweet mixture produced by bees (Apis mellifera) from the nectar of flowers, from secretions of parts of the living plants or excretions of plant-sucking insects on the living part of plants that the honey bees collect, transform and combine with specific substances of their own (such as enzymes), deposit, dehydrate, store and leave in the beeswax honeycombs to ripen and mature (Codex Alimentarius, 2010). Various physical types (pressed, centrifuged and drained) and forms (comb, chunk, crystallized or granulated, creamed and heat-processed) of honey are on the market (Crane, 1990). According to the definition of the Codex Alimentarius and other international honey standards honey shall not have any added food ingredient than honey to it nor shall any particular constituent be removed from it.

Adulteration usually refers to mixing other matters (substance(s) of an inferior and sometimes harmful quality with food or drink that contains naturally similar substance (Cordella, 2003; Pilizota and Tiban, 2009). Adulteration of honey with cheaper sweetening materials has been widely reported in various literatures (Martin et al., 1997). Honey adulteration appeared on the world market since 1970s when high-fructose corn syrup was introduced by the industry (Pilizota and Tiban, 2009). Recently honey is ranked third in the list of globally adulterated products (Zhou et al., 2018). Due to the superior nutritional and health value and unique flavor, natural honey is preferred by consumers; hence, the price of bee honey is much greater than other sweetening commodities, such as refined cane sugar and corn syrup. The high price of natural honey encourages workers in the honey industry, including beekeepers and merchants, to adulterate honey worldwide, which leads to deterioration of honey quality, but increases honey quantity that is sold at the same price of natural authentic honey (Mouazen and AlWalaan, 2014). Other adulterating materials such as molasses inverted by acids or enzymes from corn, sugar beet and syrups of natural origin such as maple (Pilizota and Tiban, 2009); banana, corn flour and materials like soil had been reported (Meseret and Taye, 2017).

In Ethiopia also, honey adulteration has become a serious problem affecting both the local and the export market of the country (Gemechis, 2016). Problems of honey fraud negatively influence market 
growth by damaging consumer confidence (Cabañero et al.2006). Recent report by (Meseret and Taye, 2017 ) indicated that about $60 \%$ of honey merchants claimed they bought adulterated honey, the major (about 69\%) adulterant material being sugar though other materials were listed too. However, the study conducted before did not include the chemical analysis of adulterated and pure honey, except undertaking some observations of physical changes adulterated honey displays. However, to guarantee authenticity of honey and protect the consumer from commercial exploitation, the quality of honey must be controlled analytically (Mendes et al., 1998). The European Commission encourages the use of analytical methods to determine the authenticity of honeys (Morales et al., 2008). Therefore, this research work aims to characterize honey adulterated with different major adulterants based on specific physical properties and chemical compositions acquired with conventional analytical methods.

\section{Materials AND Methods}

\subsection{Sample Preparation}

The major known adulterant materials were prepared in different percent (ratios) with pure honey in laboratory. These major adulterant materials were identified previously (Meseret and Taye, 2017). The major adulterant materials identified in the country were commercial sugar, banana, molasses, sheb (shebeb). Three replicates were taken to determine the chemical properties of each adulterated honey samples. The analyses were conducted in Holeta Bee Research Center and the percentage of honey with major identified and known honey adulterants were prepared as follows.

Table1. Ratio of adulterants to honey

\begin{tabular}{|c|c|c|c|c|c|c|c|c|}
\hline $\begin{array}{c}\text { Adulteran } \\
\text { ts with } \\
\text { honey }\end{array}$ & \multicolumn{7}{|c|}{ Ratios of adulterants to honey (treatments) } \\
\hline $\begin{array}{c}\text { Honey: } \\
\text { sugar }\end{array}$ & $0: 1$ & $1: 1$ & $1: 2$ & $2: 1$ & $1: 0.5$ & $5: 1$ & $10: 1$ & $20: 1$ \\
\hline $\begin{array}{c}\text { Honey: } \\
\text { sugar: } \\
\begin{array}{c}\text { water: } \\
\text { sheb }\end{array}\end{array}$ & $4: 4: 1: 0.1$ & $2: 2: 0.5: 0$ & $3: 4: 1: 0.1$ & $\begin{array}{c}5: 2: 0.5: 0 . \\
15\end{array}$ & $\begin{array}{c}4: 2: 0.5: 0 . \\
25\end{array}$ & $\begin{array}{c}1: 2: 0.5: 0 . \\
25\end{array}$ & $\begin{array}{c}2: 2: 0.5: 0 \\
.3\end{array}$ & $\begin{array}{c}2: 1: 1: 0 . \\
4\end{array}$ \\
\hline $\begin{array}{c}\text { Honey: } \\
\text { molasses }\end{array}$ & $0: 1$ & $1: 1$ & $2: 1$ & $1: 2$ & 1.5 & $5: 1$ & $10: 1$ & $20: 1$ \\
\hline $\begin{array}{c}\text { Honey: } \\
\text { banana : } \\
\text { molasses }\end{array}$ & $0: 1: 1$ & $1: 1: 1$ & $4: 1: 1$ & $1: 2: 2$ & $2: 1: 2$ & $10: 1: 1$ & $20: 2: 1$ & \\
\hline $\begin{array}{c}\text { Honey } \\
\text { sugar: } \\
\text { banana }\end{array}$ & $0: 1: 1$ & $1: 1: 1$ & $0.25: 0.25$ & $0.5: 1: 1$ & $0.25: 1: 1$ & $1: 0.5: 1$ & $10: 1: 1$ & $20: 1: 1$ \\
\hline $\begin{array}{c}\text { Honey: } \\
\text { banana: } \\
\text { molasses }\end{array}$ & $0: 1: 2.5: 0$ & $2: 1: 2.5: 0$ & $1 .: 0.5: 4: 1$ & $1: 1: 2: 1$ & $0.5: 1: 2: 0$. & $1: 0.5: 1.5:$ & $10: 1: 1: 1$ & $20: 1: 1:$ \\
\hline
\end{tabular}

\subsection{Physiochemical Properties}

\subsubsection{Colour}

Colors of the pure and adulterated honey samples were measured using Pfund honey color grader (Koehler Bohemia. NY). Hundred grams (100 g) of adulterated honey was poured into the sample holder of the Pfund grader. Determination was based on the matching of the honey sample colors with the color indexes present in the glass Pfund grader. Color of pure honey was determined in the same way and compared with that of adulterated honey.

\subsubsection{Moisture}

Moisture content was determined using Abbe refractometer (ABBE- 5 Bellingham Stanley. Ltd, United Kingdom) at $20^{\circ} \mathrm{C}$. Directly after homogenization of honey, the surface of the prism was evenly covered with the sample of honey and after 2 minutes the reading of the refractive index was recorded. Distilled water (1.3330) was used as a reference. The refractive index reading was converted to moisture content (g/100 g) using (AOAC, 1990). 


\subsubsection{Hydroxymethylfurfural}

Hydroxymethylfurfural (HMF) content was determined using $6800 \mathrm{UV}-\mathrm{Vis}$ spectrophotometer (JENWAY, United Kingdom). Different reagents were prepared as follow: Carrez solution I was prepared by weighing $15 \mathrm{~g}$ of potassium hexacyanoferrate (II), $\mathrm{K}_{4} \mathrm{Fe}(\mathrm{CN}){ }^{\bullet} 3 \mathrm{H}_{2} \mathrm{O}$ and dissolving it in water. Then, the solution was made up to $100 \mathrm{ml}$. Carrez solution II, $30 \mathrm{~g}$ of zinc acetate, $\mathrm{Zn}\left(\mathrm{CH}_{3} . \mathrm{COO}\right)_{2} .2 \mathrm{H}_{2} \mathrm{O}$ was diluted and made up to $100 \mathrm{ml}$., $0.20 \mathrm{~g}$ of solid sodium hydrogen sulphite, $\mathrm{NaHSO}_{3}$, diluted to $100 \mathrm{ml}$. Carrez solution is clarification reagents to remove these interfering compounds from the analytes. Five gram $(5 \mathrm{~g})$ of honey was weighed into a $50 \mathrm{ml}$ beaker and dissolved the sample in $25 \mathrm{ml}$ of water and transferred quantitatively into a $50 \mathrm{ml}$ volumetric flask. A $0.5 \mathrm{ml}$ of Carrez solution I was added with $0.5 \mathrm{ml}$ of Carrez solution II, mixed well and made up to the mark with water and filtered through filter paper (general purpose); the first $10 \mathrm{ml}$ of the filtrate was rejected. Five milliliters was pipetted in each of two 2 test tubes and $5 \mathrm{ml}$ of water was added to one of the test tubes and mixed well (the sample solution). Five milliliters of sodium bisulphite solution, $0.2 \%$ was added to the second test tube and mixed well. By subtracting the absorbance measured at $284 \mathrm{~nm}$ for HMF in the honey sample solution against the absorbance of reference (the same honey solution treated with sodium bisulphite, $0.2 \%$ ) at $336 \mathrm{~nm}$ as described in harmonized method (AOAC, 1990) method. HMF content of honey was calculated using the following equation:

$\mathrm{HMF}$ in $\mathrm{mg} / \mathrm{kg}$ honey $=\left(\mathrm{A}_{284}-\mathrm{A}_{336}\right) \times 149.7 \times 5 \times \mathrm{D} / \mathrm{W}$

Where A284= absorbance at $284 \mathrm{~nm}$, A336 $=$ absorbance at $336 \mathrm{~nm}$, Factor $=149.7=(126 / 1683)$ $(1000 / 10)(1000 / 5), 126=$ molecular weight of HMF, 16,830 = molar absorptive of HMF at $284 \mathrm{~nm}$, $1000=$ conversion of $\mathrm{g}$ into $\mathrm{mg}, 10=$ conversion of 5 into $50 \mathrm{~mL}, 1000=$ conversion $\mathrm{g}$ honey into 1000 $\mathrm{g}, 5=$ nominal sample weight, $\mathrm{D}=$ Dilution factor and $\mathrm{W}=$ weight in $\mathrm{g}$ of the honey sample

\subsubsection{Free Acidity and $P H$}

Free acidity of honey is the content of all free acids, expressed in milli equivalents per kilogram honey (meq of acid $/ \mathrm{kg}$ ) was determined using $\mathrm{pH}$ meter (METTLER TOLEDO, CHINA) and titrating until $\mathrm{pH}$ value of 8.3. Ten gram of honey sample was dissolved in $75 \mathrm{ml}$ distilled water in $250 \mathrm{ml}$ volumetric flask and the standardized $0.1 \mathrm{M} \mathrm{NaOH}$ and the dissolved honey sample was titrated with standardized $0.1 \mathrm{M} \mathrm{NaOH}$ to $\mathrm{pH} 8.3$ using $\mathrm{pH}$ glass electrode attached to $\mathrm{pH}$ meter as end point indicator. The $\mathrm{pH}$ value was determined by using glass electrode after calibration with standard buffer solution $\mathrm{pH} \mathrm{4,7}$ and 10 (AOAC, 1990) method.

\subsubsection{Ash}

Ten gram of the honey samples $\left(\mathrm{m}_{0}\right)$ was weighed into a pre-weighed crucible $\left(\mathrm{m}_{2}\right)$ and two drops of olive oil was added to the honey sample. Then, ash dish $\left(\mathrm{m}_{1}\right)$ with honey was placed on hotplate to remove water from the honey at low heat rising to $350-400^{\circ} \mathrm{C}$ and after the preliminary ashing, and the dish was placed in the preheated a muffle furnace at $600^{\circ} \mathrm{C}$ until ashing complete (Bio Base JKKZ .5.12GJ Muffle Furnace, Shandong. ldt, China) and was heated for 1 hour and the ash dish cooled down in the desiccators $\left(\mathrm{m}_{1}\right)$ and weighed as described in harmonized IHC (Bogdanov, 2009). The proportion of ash $\left(\mathrm{W}_{\mathrm{A}}\right)$ in $\mathrm{g} / 100 \mathrm{~g}$ honey was calculated using the following formula:

$\left.\mathrm{W}_{\mathrm{A}}=\left(\mathrm{m}_{1}-\mathrm{m}_{2}\right) / \mathrm{m}_{0}\right) * 100$

Where: $\mathrm{m}_{0}=$ weight of honey sample, $\mathrm{m}_{1}=$ weight of crucible with ash, $\mathrm{m}_{2}=$ weight of dish .

\subsubsection{Electrical Conductivity}

The electrical conductivity of a solution of $20 \mathrm{~g}$ dry matter of honey in $100 \mathrm{ml}$ distilled water was measured using an electrical conductivity cell (BANTE Instrument- 520 conductive and temperature meter, China). A $0.745 \mathrm{~g}$ of potassium chloride, was dried at $130^{\circ} \mathrm{C}$, dissolved in freshly distilled water in a $100 \mathrm{ml}$ flask and filled to volume with distilled water. Forty milliliters of the potassium chloride solution was transferred to a beaker and the conductivity cell connected to the conductivity meter, the cell was rinsed thoroughly with potassium chloride solution and immersed in the solution, together with a thermometer and reading of the electrical conductance of the solution in $\mathrm{mS}$ after the temperature equilibrated to $20^{\circ} \mathrm{C}$ was taken as described in harmonized IHC (Bogdanov, 2009). The cell constant $\mathrm{K}$, was calculated using the following formula:

$\mathrm{K}=11.691 \times 1 / \mathrm{G}$ 
Where: $\mathrm{K}=$ the cell constant in $\mathrm{cm}^{-1}, \mathrm{G}=$ the electrical conductance in $\mathrm{mS}$, measured with the conductivity cell, 11.691= the sum of the mean value of the electrical conductivity of freshly distilled water in $\mathrm{mS}_{\mathrm{cm}} \mathrm{cm}^{-1}$ and the electrical conductivity of a $0.1 \mathrm{M}$ potassium chloride solution, at $20^{\circ} \mathrm{C}$.

\section{RESULTS AND DISCUSSION}

\subsection{Color}

The color of honey is a useful parameter for the characterization of the product. Color is the single most important factor determining import and wholesale prices (Belay et al., 2015). The color of the honey and sugar adulteration, grouped as dark to light amber, the adulterated honey with the sugar:water and sheb color were light amber, the color of adulterated honey from honey and molasses amber to dark, adulterated honey from honey : banana : molasses was found to be amber to dark and adulterated honey prepared from honey : sugar: banana color found to be white. The variation among the adulterated honey types was due to the adulterating material of the honey. The color of the honey samples from the various regions could not be the single determinant of adulteration.

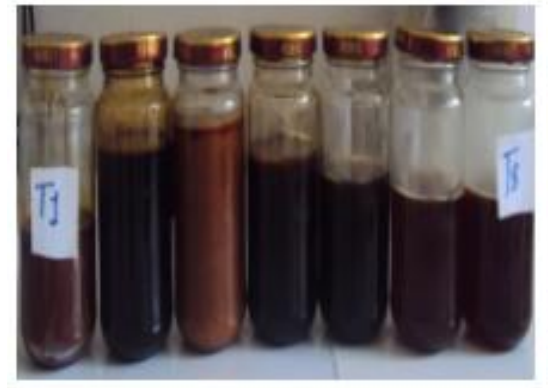

Figure1. Honey: sugar

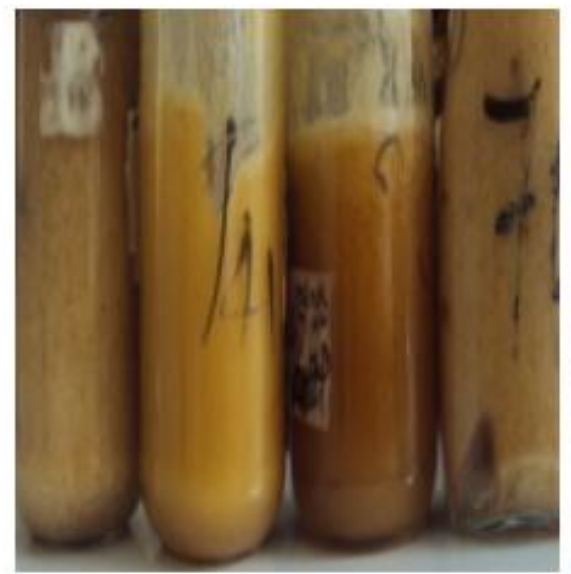

Figure3. Honey: sugar: banana

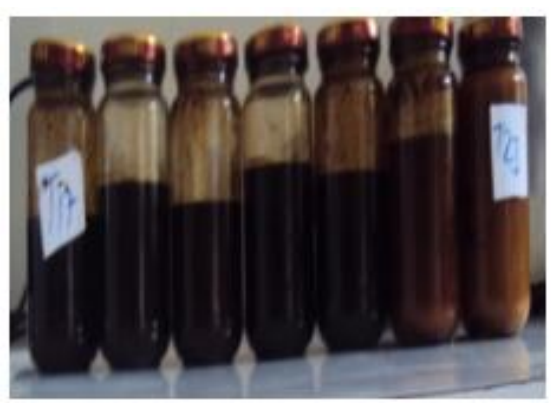

Figure 2. Honey: molasses

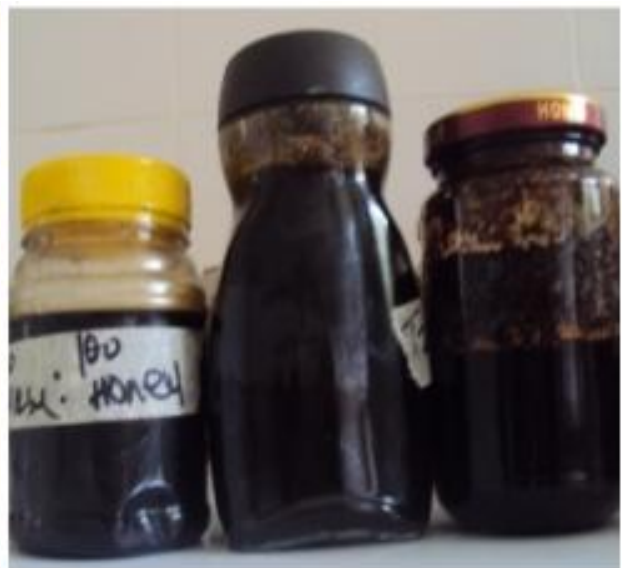

Figure4. Honey: banana: molasses

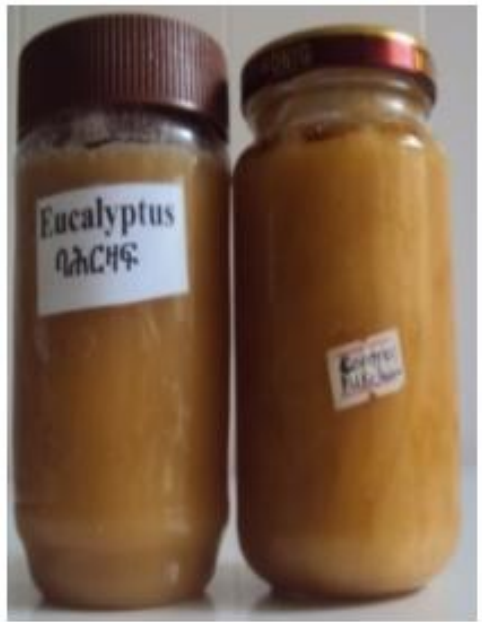

Figure5. Pure honey

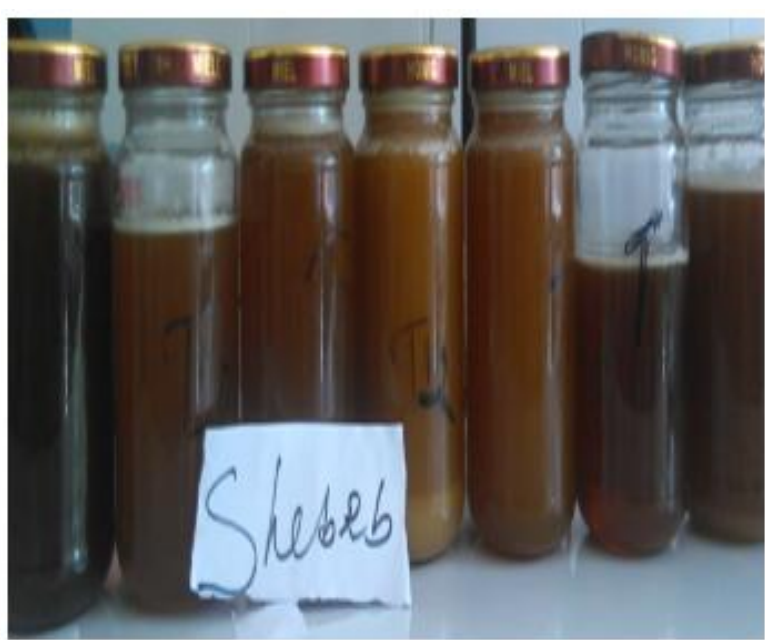

Figure6. Honey: Sugar: water: sheb (shebeb) 
Chemical and Physical Properties of Adulterated Honey and Developing Means of Identifying Adulterants

Table2. Analytical results of honey samples adulterated with sugar, water and sheb by different ratio

\begin{tabular}{|c|c|c|c|c|c|c|}
\hline \multirow[t]{2}{*}{ Treatment } & \multicolumn{6}{|c|}{ Parameters $($ mean \pm SE $)$} \\
\hline & $\begin{array}{c}\mathrm{HMF} \\
(\mathrm{mg} / \mathrm{kg})\end{array}$ & $\mathrm{pH}$ & $\begin{array}{c}\mathrm{EC} \\
(\mathrm{mS} / \mathrm{cm})\end{array}$ & Ash (\%) & $\begin{array}{l}\text { Free Acidity } \\
(\mathrm{meq} / \mathrm{kg})\end{array}$ & $\overline{\mathrm{MC}(\%)}$ \\
\hline $\begin{array}{c}\text { Honey:sugar:water:sheb } \\
(4: 4: 1: 0.1)\end{array}$ & $0.00 \pm 0.00^{\mathrm{d}}$ & $3.25 \pm 0.16^{\mathrm{b}}$ & $0.83 \pm 0.06^{\mathrm{b}}$ & $0.39 \pm 0.03^{\mathrm{b}}$ & $161.00 \pm 10.54^{\mathrm{b}}$ & $14.5 \pm 0.00^{\mathrm{de}}$ \\
\hline $\begin{array}{c}\text { Honey:sugar:water:sheb } \\
(2: 2: 0.5: 0.1)\end{array}$ & $0.00 \pm 0.00^{\mathrm{d}}$ & $3.24 \pm 0.13^{b}$ & $0.80 \pm 0.35^{\mathrm{b}}$ & $0.38 \pm 0.20^{\mathrm{b}}$ & $110.33 \pm 19.46^{\mathrm{b}}$ & $14.0 \pm 0.28^{\mathrm{de}}$ \\
\hline $\begin{array}{c}\text { Honey:sugar:water:sheb } \\
(3: 4: 1: 0.1)\end{array}$ & $0.00 \pm 0.00^{\mathrm{d}}$ & $3.39 \pm 0.15^{\mathrm{b}}$ & $0.59 \pm 0.09^{\mathrm{b}}$ & $0.25 \pm 0.05^{\mathrm{b}}$ & $102.33 \pm 5.24^{\mathrm{b}}$ & $13.0 \pm 0.28^{\mathrm{f}}$ \\
\hline $\begin{array}{c}\text { Honey:sugar:water::sheb } \\
(5: 2: 0.5: 0.15)\end{array}$ & $23 \pm 8.03^{b}$ & $3.26 \pm 0.15^{\mathrm{b}}$ & $1.17 \pm 0.02^{\mathrm{ab}}$ & $0.51 \pm 0.01^{\mathrm{ab}}$ & $168.33 \pm 5.24^{\mathrm{b}}$ & $14.9 \pm 0.10^{\mathrm{cd}}$ \\
\hline $\begin{array}{c}\text { Honey:sugar:water:sheb } \\
(4: 2: 0.5: 0.2)\end{array}$ & $35.7 \pm 8.20^{\mathrm{a}}$ & $3.18 \pm 0.16^{\mathrm{b}}$ & $1.33 \pm 0.09^{\mathrm{ab}}$ & $0.68 \pm 0.05^{\mathrm{ab}}$ & $212.33 \pm 6.22^{\mathrm{b}}$ & $15.6 \pm 0.16^{\mathrm{bc}}$ \\
\hline $\begin{array}{c}\text { Honey:sugar:water:sheb } \\
(1: 2: 0.5: 0.25)\end{array}$ & $0.00 \pm 0.00^{\mathrm{d}}$ & $3.03 \pm 0.05^{\mathrm{b}}$ & $0.88 \pm 0.26^{\mathrm{b}}$ & $0.42 \pm 0.14^{\mathrm{b}}$ & $452.66 \pm 6.74 \mathbf{a}$ & $14.4 \pm 0.21^{\mathrm{de}}$ \\
\hline $\begin{array}{c}\text { Honey:sugar:water:sheb } \\
(2: 2: 0.5: 0.3)\end{array}$ & $0.00 \pm 0.00^{\mathrm{d}}$ & $3.26 \pm 0.11^{\mathrm{b}}$ & $1.94 \pm 0.71^{\mathrm{a}}$ & $1.03 \pm 0.40^{\mathrm{a}}$ & $458.33 \pm 1.66^{\mathrm{a}}$ & $13.9 \pm 0.16^{\mathrm{ef}}$ \\
\hline $\begin{array}{c}\text { Honey:sugar:water:sheb } \\
(2: 1: 1: 0.4)\end{array}$ & $0.00 \pm 0.00^{\mathrm{d}}$ & $3.27 \pm 0.12^{b}$ & $1.09 \pm 0.38^{\mathrm{ab}}$ & $0.54 \pm 0.21^{\mathrm{ab}}$ & $252.33 \pm 112.3^{\mathrm{b}}$ & $19.1 \pm 0.10^{\mathrm{a}}$ \\
\hline Pure honey & $9.96 \pm 0.12^{\mathrm{c}}$ & $4.46 \pm 0.04^{\mathrm{a}}$ & $0.69 \pm 0.02^{\mathrm{b}}$ & $0.47 \pm 0.03^{\mathrm{ab}}$ & $14.66 \pm 2.18^{c}$ & $16.02 \pm 0.26^{\mathrm{a}}$ \\
\hline Ethiopian honey standard & $\leq 40$ & & $\leq 0.8$ & $\leq 0.6$ & $\leq 40$ & $\leq 21$ \\
\hline
\end{tabular}

Different letters down column showed significant difference $(p<0.05)$

Table3. Test results of honey samples adulterated with molasses by different ratio

\begin{tabular}{|c|c|c|c|c|c|c|}
\hline \multirow{2}{*}{ Treatment } & \multicolumn{5}{|c|}{ Parameters (mean \pm SE) } \\
\cline { 2 - 7 } & $\begin{array}{c}\text { HMF } \\
(\mathrm{mg} / \mathrm{kg})\end{array}$ & $\mathrm{pH}$ & $\begin{array}{c}\text { EC } \\
(\mathrm{mS} / \mathrm{cm})\end{array}$ & FA (meq/kg) & Ash $(\%)$ & MC $(\%)$ \\
\hline $\begin{array}{c}\text { Honey:molasses } \\
(0: 1)\end{array}$ & $49.63 \pm 1.16^{\mathrm{c}}$ & $5.80 \pm 0.00^{\mathrm{a}}$ & $8.23 \pm 7.7^{\mathrm{ab}}$ & $164.77 \pm 2.90^{\mathrm{a}}$ & $4.65 \pm 4.4^{\mathrm{ab}}$ & $7.27 \pm 1.23^{\mathrm{e}}$ \\
\hline $\begin{array}{c}\text { Honey:molasses } \\
(1: 1)\end{array}$ & $0.00 \pm 0.00^{\mathrm{e}}$ & $5.59 \pm 0.05^{\mathrm{bc}}$ & $8.52 \pm 1.85^{\mathrm{ab}}$ & $107.00 \pm 5.13^{\mathrm{bc}}$ & $4.81 \pm 1.06 \mathrm{ab}$ & $14.31 \pm 1.81^{\mathrm{d}}$ \\
\hline $\begin{array}{c}\text { Honey:molasses } \\
(2: 1)\end{array}$ & $0.00 \pm 0.00^{\mathrm{e}}$ & $5.38 \pm 0.09^{\mathrm{abc}}$ & $6.98 \pm 5.4^{\mathrm{ab}}$ & $86.00 \pm 9.00^{\mathrm{cd}}$ & $3.93 \pm 3.13^{\mathrm{ab}}$ & $19.83 \pm 0.72^{\mathrm{b}}$ \\
\hline $\begin{array}{c}\text { Honey:molasses } \\
(1: 2)\end{array}$ & $19.6 \pm .10 .1^{\mathrm{d}}$ & $5.66 \pm 0.06^{\mathrm{ab}}$ & $15.83 \pm 1.3^{\mathrm{a}}$ & $127.33 \pm 2.66^{\mathrm{b}}$ & $9.04 \pm 0.79^{\mathrm{a}}$ & $14.63 \pm 0.39^{\mathrm{d}}$ \\
\hline $\begin{array}{c}\text { Honey:molasses } \\
(1: 5)\end{array}$ & $130.3 \pm .88^{\mathrm{a}}$ & $5.53 \pm 0.03^{\mathrm{abc}}$ & $7.96 \pm 0.18^{\mathrm{ab}}$ & $90.77 \pm 4.33^{\mathrm{c}}$ & $4.49 \pm 0.46^{\mathrm{ab}}$ & $14.35 \pm 0.08^{\mathrm{d}}$ \\
\hline $\begin{array}{c}\text { Honey:molasses } \\
(5: 1)\end{array}$ & $86.5 \pm 3.75^{\mathrm{b}}$ & $4.58 \pm 0.59^{\mathrm{bc}}$ & $9.10 \pm 2.14 \mathrm{ab}$ & $64.77 \pm .33^{\mathrm{de}}$ & $5.15 \pm 1.23^{\mathrm{ab}}$ & $19 \pm 0.00 \mathrm{~b}^{\mathrm{c}}$ \\
\hline $\begin{array}{c}\text { Honey:molasses } \\
(10: 1)\end{array}$ & $0.00 \pm 0.00 \mathrm{e}$ & $4.70 \pm 0.12^{\mathrm{bc}}$ & $9.32 \pm 3.7^{\mathrm{ab}}$ & $49.33 \pm 1.76^{\mathrm{ef}}$ & $5.27 \pm 2.16^{\mathrm{ab}}$ & $17.76 \pm 0.14^{\mathrm{bcd}}$ \\
\hline $\begin{array}{c}\text { Honey:mollases } \\
(20: 1)\end{array}$ & $0.00 \pm 0.00^{\mathrm{e}}$ & $4.54 \pm 0.11^{\mathrm{c}}$ & $1.31 \pm 0.40^{\mathrm{ab}}$ & $40.00 \pm 5.03^{\mathrm{f}}$ & $0.67 \pm 0.23^{\mathrm{b}}$ & $24.16 \pm 0.29^{\mathrm{a}}$ \\
\hline $\begin{array}{c}\text { Pure honey } \\
\text { Ethiopian } \\
\text { honey } \\
\text { standard }\end{array}$ & $9.96 \pm 0.12^{\mathrm{de}}$ & $4.46 \pm 0.04^{\mathrm{c}}$ & $0.69 \pm 0.02^{\mathrm{b}}$ & $14.66 \pm 2.18$ & $0.47 \pm 0.03^{\mathrm{b}}$ & $16.02 \pm 0.26^{\mathrm{cd}}$ \\
\hline
\end{tabular}

Different letters down column showed significant difference $(p<0.05)$

Table4. Test results of honey samples adulterated with sugar and banana by different ratio

\begin{tabular}{|c|c|c|c|c|c|c|}
\hline \multirow{2}{*}{ Treatment } & \multicolumn{5}{|c|}{ Parameters (mean \pm SE) } & Ash $\%)$ \\
\cline { 2 - 6 } & $\begin{array}{c}\text { HMF } \\
(\mathrm{mg} / \mathrm{kg})\end{array}$ & $\mathrm{pH}$ & $\begin{array}{c}\text { FA } \\
(\mathrm{meq} / \mathrm{kg})\end{array}$ & MC $(\%)$ \\
\hline $\begin{array}{c}\text { Honey } \\
\text { :sugar:banana } \\
(0: 1: 1)\end{array}$ & $0.00 \pm 0.00^{\mathrm{b}}$ & $4.9 \pm 0.10^{\mathrm{ab}}$ & $0.43 \pm 0.12^{\mathrm{ab}}$ & $0.90 \pm 0.22^{\mathrm{ab}}$ & $33.66 \pm 2.18^{\mathrm{ab}}$ & $23.23 \pm 1.6^{\mathrm{bc}}$ \\
\hline $\begin{array}{c}\text { Honey:sugar:banana } \\
(1: 1: 1)\end{array}$ & $0.00 \pm 0.00^{\mathrm{b}}$ & $4.52 \pm 0.12^{\mathrm{bc}}$ & $0.40 \pm 0.12^{\mathrm{ab}}$ & $0.84 \pm 0.20^{\mathrm{ab}}$ & $35.00 \pm 0.57^{\mathrm{ab}}$ & $18.61 \pm 0.59^{\mathrm{de}}$ \\
\hline $\begin{array}{c}\text { Honey:sugar:banana } \\
(0.25: 0.25: 1)\end{array}$ & $0.00 \pm 0.00^{\mathrm{b}}$ & $4.50 \pm 0.17^{\mathrm{bc}}$ & $0.36 \pm 0.14^{\mathrm{b}}$ & $0.77 \pm 0.24^{\mathrm{b}}$ & $41.66 \pm 4.84^{\mathrm{ab}}$ & $24.8 \pm 0.86^{\mathrm{bc}}$ \\
\hline
\end{tabular}


Chemical and Physical Properties of Adulterated Honey and Developing Means of Identifying Adulterants

\begin{tabular}{|c|c|c|c|c|c|c|}
\hline $\begin{array}{c}\text { Honey:sugar:banana } \\
(0.5: 1: 1)\end{array}$ & $0.00 \pm 0.00^{\mathrm{b}}$ & $5.30 \pm 0.18^{\mathrm{a}}$ & $3.08 \pm 1.53^{\mathrm{a}}$ & $5.50 \pm 2.66^{\mathrm{a}}$ & $66.33 \pm 16.8^{\mathrm{a}}$ & $38.27 \pm 0.14^{\mathrm{a}}$ \\
\hline $\begin{array}{c}\text { Honey:sugar:banana } \\
(0.25: 1: 1)\end{array}$ & $0.00 \pm 0.00^{\mathrm{b}}$ & $4.70 \pm 0.14^{\mathrm{ab}}$ & $0.37 \pm 0.04^{\mathrm{b}}$ & $0.79 \pm 0.07^{\mathrm{b}}$ & $\begin{array}{c}39.33 \pm 5.17 \\
\mathrm{ab}\end{array}$ & $19.45 \pm 0.93^{\mathrm{cd}}$ \\
\hline $\begin{array}{c}\text { Honey:sugar:banana } \\
(1: 0.5: 1)\end{array}$ & $0.00 \pm 0.00^{\mathrm{b}}$ & $4.57 \pm 0.13^{\mathrm{abc}}$ & $0.52 \pm .08^{\mathrm{ab}}$ & $1.04 \pm 0.14^{\mathrm{ab}}$ & $39.00 \pm 1.15^{\mathrm{ab}}$ & $26.23 \pm 0.48^{\mathrm{b}}$ \\
\hline $\begin{array}{c}\text { Honey:sugar:banana } \\
(10: 1: 1)\end{array}$ & $2.46 \pm 0.15^{\mathrm{b}}$ & $4.03 \pm 0.21^{\mathrm{c}}$ & $0.65 \pm 0.16^{\mathrm{ab}}$ & $\begin{array}{c}1.27 \pm 0.28 \\
\mathrm{ab}\end{array}$ & $28.66 \pm 0.88^{\mathrm{b}}$ & $24.53 \pm 0.16^{\mathrm{bc}}$ \\
\hline $\begin{array}{c}\text { Honay : } \\
\text { sugar:banana } \\
(20: 1: 1)\end{array}$ & $5.53 \pm 0.28^{\mathrm{ab}}$ & $4.05 \pm 0.18^{\mathrm{c}}$ & $0.17 \pm 0.01^{\mathrm{b}}$ & $0.43 \pm 0.01^{\mathrm{b}}$ & $38.33 \pm 7.75^{\mathrm{ab}}$ & $24.33 \pm 0.33^{\mathrm{cd}}$ \\
\hline Pure honey & $9.96 \pm 0.12^{\mathrm{a}}$ & $4.46 \pm 0.04^{\mathrm{bc}}$ & $0.47 \pm 0.03^{\mathrm{b}}$ & $0.69 \pm 0.02^{\mathrm{b}}$ & $14.66 \pm 2.18^{\mathrm{b}}$ & $16.02 \pm 0.02^{\mathrm{e}}$ \\
\hline $\begin{array}{c}\text { Ethiopian honey } \\
\text { standard }\end{array}$ & $\leq 40$ & & $\leq 0.6$ & $\leq 0.8$ & $\leq 40$ & $\leq 21$ \\
\hline
\end{tabular}

Different letters down the column showed significant difference $(p<0.05)$

Table5. Test results of honey samples adulterated with sugar

\begin{tabular}{|c|c|c|c|c|c|c|}
\hline \multirow{2}{*}{ Treatment } & \multicolumn{7}{|c|}{ Parameters mean \pm SE } \\
\cline { 2 - 7 } & $\begin{array}{c}\text { HMF } \\
(\mathrm{mg} / \mathrm{kg})\end{array}$ & $\mathrm{P}^{\mathrm{H}}$ & Ash $(\%)$ & $\begin{array}{c}\text { EC } \\
(\mathrm{mS} / \mathrm{cm})\end{array}$ & $\begin{array}{c}\text { FA } \\
(\mathrm{meq} / \mathrm{kg})\end{array}$ & MC $(\%)$ \\
\hline Honey:sugar (1:1) & $0.00 \pm 0.00^{\mathrm{b}}$ & $3.21 \pm 0.02^{\mathrm{c}}$ & $0.04 \pm 0.01^{\mathrm{b}}$ & $0.19 \pm 0.01^{\mathrm{c}}$ & $78.66 \pm 0.66^{\mathrm{a}}$ & $13 \pm 0.28^{\mathrm{e}}$ \\
\hline Honey:sugar (2:4) & $47.1 \pm 6.23^{\mathrm{a}}$ & $3.74 \pm 0.03^{\mathrm{b}}$ & $0.20 \pm 0.05^{\mathrm{b}}$ & $\begin{array}{c}0.48 \pm 0.10 \\
\mathrm{~b}\end{array}$ & $36.33 \pm 5.23^{\mathrm{b}}$ & $13.5 \pm 0.29^{\mathrm{cd}}$ \\
\hline Honey:sugar (5:1) & $0.00 \pm 0.00^{\mathrm{b}}$ & $3.86 \pm 0.11^{\mathrm{b}}$ & $0.17 \pm 0.02^{\mathrm{b}}$ & $0.44 \pm 0.04^{\mathrm{b}}$ & $32.00 \pm 4.16^{\mathrm{b}}$ & $13.16 \pm 0.16^{\mathrm{e}}$ \\
\hline Honey:sugar (10:1) & $1.36 \pm 1.36^{\mathrm{ab}}$ & $4.04 \pm 0.14^{\mathrm{ab}}$ & $0.22 \pm 0.02^{\mathrm{b}}$ & $0.52 \pm 0.04^{\mathrm{ab}}$ & $27.66 \pm 2.33^{\mathrm{bc}}$ & $14.33 \pm 0.16^{\mathrm{bc}}$ \\
\hline Honey:sugar (20:1) & $5.63 \pm 5.28^{\mathrm{ab}}$ & $4.06 \pm 0.17^{\mathrm{ab}}$ & $0.12 \pm 0.05^{\mathrm{b}}$ & $0.35 \pm 0.11^{\mathrm{bc}}$ & $28.66 \pm 2.40^{\mathrm{bc}}$ & $15 \pm 0.00^{\mathrm{bb}}$ \\
\hline Pure honey & $9.96 \pm 0.12^{\mathrm{b}}$ & $4.46 \pm 0.04^{\mathrm{a}}$ & $0.47 \pm 0.03^{\mathrm{a}}$ & $0.69 \pm 0.02^{\mathrm{a}}$ & $14.66 \pm 2.18^{\mathrm{c}}$ & $16.02 \pm 0.26^{\mathrm{a}}$ \\
\hline $\begin{array}{c}\text { Ethiopian honey } \\
\text { standard }\end{array}$ & $\leq 40$ & & $\leq 0.6$ & $\leq 0.8$ & $\leq 40$ & $\leq 21$ \\
\hline
\end{tabular}

Different letters down the column showed significant difference $(p<0.05)$

Table6. Test results of honey samples adulterated with banana and molasses

\begin{tabular}{|c|c|c|c|c|c|c|}
\hline & \multicolumn{5}{|c|}{ Parameters (mean \pm SE) } \\
\cline { 2 - 7 } $\begin{array}{c}\text { Treatment } \\
\text { HMF } \\
(\mathrm{mg} / \mathrm{kg})\end{array}$ & $\mathrm{pH}$ & Ash $(\%)$ & EC $(\mathrm{mS} / \mathrm{cm})$ & FA $(\mathrm{meq} / \mathrm{kg})$ & MC $(\%)$ \\
\hline $\begin{array}{c}\text { Honey:banana:molasses } \\
(0: 1: 1)\end{array}$ & $35.33 \pm 11.04^{\mathrm{a}}$ & $5.64 \pm 0.06^{\mathrm{a}}$ & $4.02 \pm 1.99^{\mathrm{ab}}$ & $7.14 \pm 3.74^{\mathrm{ab}}$ & $\begin{array}{c}102.33 \pm 18.26 \\
\text { ab }\end{array}$ & $58.6 \pm 2.7^{\mathrm{a}}$ \\
\hline $\begin{array}{c}\text { Honey:banana:molasses } \\
(1: 1: 1)\end{array}$ & $0.00 \pm 0.00^{\mathrm{b}}$ & $5.42 \pm 0.07^{\mathrm{ab}}$ & $2.69 \pm 1.01^{\mathrm{ab}}$ & $4.82 \pm 1.75^{\mathrm{abc}}$ & $78 \pm 5.68^{\mathrm{abcd}}$ & $53.09 \pm 3.63^{\mathrm{a}}$ \\
\hline $\begin{array}{c}\text { Honey:banana:molasses } \\
(4: 1: 1)\end{array}$ & $0.00 \pm 0.00^{\mathrm{b}}$ & $5.19 \pm 0.07^{\mathrm{bc}}$ & $1.05 \pm 0.05^{\mathrm{b}}$ & $1.96 \pm 0.09^{\mathrm{bc}}$ & $56 \pm 3.51^{\mathrm{bcde}}$ & $37.83 \pm 3.58^{\mathrm{b}}$ \\
\hline $\begin{array}{c}\text { Honey:banana:molasses } \\
(1: 2: 2)\end{array}$ & $0.00 \pm 0.00^{\mathrm{b}}$ & $5.55 \pm 0.05^{\mathrm{ab}}$ & $3.79 \pm 1.41^{\mathrm{ab}}$ & $6.74 \pm 2.46^{\mathrm{abc}}$ & $90.3 \pm 6.35^{\mathrm{abc}}$ & $36.90 \pm 0.26^{\mathrm{b}}$ \\
\hline $\begin{array}{c}\text { Honey:banana:molasses } \\
(2: 1: 2)\end{array}$ & $0.00 \pm 0.00^{\mathrm{b}}$ & $5.58 \pm 0.12^{\mathrm{a}}$ & $4.78 \pm 0.03^{\mathrm{a}}$ & $8.47 \pm 2.33^{\mathrm{a}}$ & $116.1 \pm 19.21^{\mathrm{a}}$ & $23.93 \pm 1.79^{\mathrm{c}}$ \\
\hline $\begin{array}{c}\text { Honey:banana:molasses } \\
(10: 1: 1\end{array}$ & $0.00 \pm 0.00^{\mathrm{b}}$ & $4.95 \pm 0.04^{\mathrm{c}}$ & $2.96 \pm 0.56^{\mathrm{ab}}$ & $5.30 \pm 0.05^{\mathrm{abc}}$ & $42.6 \pm 1.86^{\mathrm{cde}}$ & $24.13 \pm 0.18^{\mathrm{c}}$ \\
\hline $\begin{array}{c}\text { Honey:banana:molasses } \\
(20: 2: 1)\end{array}$ & $0.00 \pm 0.00^{\mathrm{c}}$ & $4.56 \pm 0.11^{\mathrm{d}}$ & $1.60 \pm 0.39^{\mathrm{ab}}$ & $2.93 \pm 0.98^{\mathrm{abc}}$ & $34.3 \pm 1.88^{\mathrm{de}}$ & $9.54 \pm 0.44^{\mathrm{d}}$ \\
\hline Pure honey & $9.96 \pm 0.12^{\mathrm{b}}$ & $4.46 \pm .04^{\mathrm{d}}$ & $0.47 \pm 0.03^{\mathrm{b}}$ & $0.69 \pm 0.02^{\mathrm{c}}$ & $14.67 \pm 2.18^{\mathrm{e}}$ & $16.02 \pm 0.26^{\mathrm{cd}}$ \\
\hline $\begin{array}{c}\text { Ethiopian honey } \\
\text { standard }\end{array}$ & $\leq 40$ & & $\leq 0.6$ & $\leq 0.8$ & $\leq 40$ & $\leq 21$ \\
\hline
\end{tabular}

Where SE is the standard deviation of each measurement values. EC is Electrical conductivity, HMF, Hydroxymethylfurfural, FA, Free Acidity and MC, Moisture Content. Different letters down the column showed significant difference $(\mathrm{p}<0.05)$

\subsection{Free Acidity}

Free acidity content indicate the freshness of the honey samples and absence of unwanted fermentation in the honey sample. In tables the free acidity content increase when adulterating 
material ratio increase which means, the pure honey free acidity content is significantly different from adulterated honey with sugar, banana, molasses, sheb and water $(\mathrm{P}<0.05)$ as showed in (Table 2$)$ indicate fermentation the honey and highest FA content observed in honey: sugar: water: sheb (2:2:0.5:0.3) treatment $458.33 \pm 1.66 \mathrm{meq} / \mathrm{kg}$ of honey. Most of adulterated samples with the highest ratio of the adulterating material free acidity content were above limit set by CA, EU and Ethiopian standards. The maximum limit for free acid set by the CA is $50 \mathrm{meq} / \mathrm{kg}$ of honey, while the EU and Ethiopian standard is $40 \mathrm{meq} / \mathrm{kg}$ of honey (Bogdanov, 2007).

\subsection{HMF Content}

The result of this study showed that there is significant difference in HMF content between the adulterated honey and pure honey and among the group of adulterating materials and ratios of adulterants (Table 2-5). The highest HMF content $(130.3 \pm 0.88 \mathrm{mg} / \mathrm{kg}$ ) was recorded in honey adulterated with molasses in ratio of 1:5 (honey to molasses) as mentioned in Table 3. However, it was observed that HMF of the adulterated honey was found to be lower than the pure honey. This might be due to the freshness or unheated or properties of the adulterating materials such as banana, sugar and water used.

\subsection{PH Value}

In general, honey is acidic in nature irrespective of its variable geographical origin. The main acid is gluconic acid. pH content of pure honey were significantly different from the adulterate honey with molasses, banana, sugar, sheb, water as showed in (Table 2-6). The high acidity of honey correlates with the fermentation of sugars present in the honey into organic acid, which is responsible for two important characteristics of honey flavor and stability against microbial spoilage (Bogdanov et al., 2008) and the

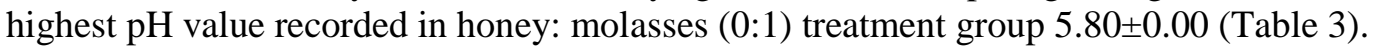

\subsection{Moisture Content}

The moisture content is an important criterion for assessing the ripeness of the honey and its shelf-life. In general, high amount of water causes the honey to ferment, to spoil and to lose flavor, with following honey quality loss. Moisture content of adulterated honeys was significantly different from pure honey at $(\mathrm{p}<0.05)$ as shown (Table 2-6) and highest moisture content was recorded on honey: banana: molasses (0:1:1) adulteration types $58.6 \pm 2.7 \%$ (Table 6) adulteration of honey with those material can increase the moisture content of honey this can lead to fermentation and spoilage of honey. An increase in moisture content of honey is indicative of adulteration the result of this study was supported by different pervious findings (Lawal et al., 2009, Kingsta et al. 2018) found that higher the adulterant added to pure honey.

\subsection{Electrical Conductivity}

The result shows that almost in all of the adulterant samples, higher conductivity records were obtained. Honey and molasses adulterated sample with different ratio found to be the highest electrical conductivity ranges $15.83 \mathrm{mS} / \mathrm{cm}$ (Table 3). Significant differences were observed between pure honey and adulterated honey treatment group (Table 2-6) and some of the results were above the limit of Ethiopian and EU standard that is greater than $0.8 \mathrm{mS} / \mathrm{cm}$. The results also had shown that in all of the adulterated honey samples the highest is the electrical conductivity has the highest ash and acid contents which agrees with that of (Bogdanov et al., 2002).

\subsection{Ash Content}

The ash content is a measure of the mineral elements in the honey samples and directly measure inorganic residue after carbonization (Lawal et al., 2009). According to the result, most of the ash contents of above the standard limit. This indicates high mineral content which could be due to adulteration because the pure honey ash content is within the standard limit, the highest ash content were obtained from adulteration of mean 9.04 $.79 \%$ honey: molasses (1:2) (Table 3) and that of pure honey were significantly different from adulterated honey as shown (Table 2-6). This result also agrees with study result of (Ribeiro et al., 2014) detection of honey adulteration of high fructose corn syrup by Low Field Nuclear Magnetic Resonance (LF 1H NMR).

\section{CONCLUSION AND RECOMMENDATION}

In current study, well known honey samples were used to distinguish pure honey from adulterated honey with molasses, sugar, banana, "sheb" and water using chemical properties. The study indicated that 
adulterated honey sample could be identified from pure honey sample by using the ash content, free acidity and electrical conductivity. However, according to this result HMF content may not identify adulteration of honey because of HMF content values affected by over heating or aging of honey. This result also showed that color parameter could not identify adulteration of honey. It can be concluded that adulteration can affect the chemical and quality properties of honey. Color parameter is not recommended for identification of adulterated honey. Therefore, users must be take care of buying fake honey based on color which is similar to pure honey but it may be adulterated honey which may affect the health and economy of the community. In addition, any honey bought from local market or mobile traders should be seen in laboratory before consuming.

\section{REFERENCES}

[1] Adgaba N, (1999). Quality state and grading of Ethiopian honey. Proceedings of the first National Conference of Ethiopian Beekeepers Association (EBA), pp. 74-82.

[2] AOAC (1990). Official Methods of Analysis of the Association of Official Analytical Chemists 15th Edition Washington D.C. USA.

[3] Bogdanov, Stefan, P. Martin, and C. Lullmann. (2002)."Harmonized methods of the international honey commission." Swiss Bee Research Centre, FAM, Liebefeld.

[4] Bogdanov, S. (2004). Quality and standards of pollen and beeswax. Apiculture, 38 (2004), 334-341.

[5] Codex Alimentarius Commission Standards (2010). Draft revised standard for honey. 19-36 (Geneve, Switzerland).

[6] Crane, E. (1990). Bees and beekeeping: science, practice and world resources. Heinnmann Newness, London. Pp 614.

[7] European Commission (2002). 2001/110/EC of 20 December 2001 relating to honey. Official Journal of the European Communities L10, 47-52.

[8] Gemechis LY (2016). Honey production and marketing in Ethiopia. Agriculture and Biology Journal of North America, 7: 248- 253.

[9] Guler, A., Bakan, A., Nisbet, C., Yavuz, O. (2007). Determination of important biochemical properties of honey to discriminate pure and adulterated honey with sucrose (Saccharum officinarum L.) syrup. Food chemistry, 105(3), 1119-1125.

[10] Harmonized methods of the International Honey Commission (2009).

[11] Mendes, E., Proenca, E. B., Ferreira, I.M.P.L.V.O. and Ferreira, M.A. (1998). Quality evaluation of Portuguese honey. Carbohydrate Polymers, 37: 219-222.

[12] Meseret Gemeda and Taye Negera (2017). Assessing the Effect of Adulteration on Honey andBeeswax Quality and Designing Way of Identification in Oromia. International Journal of Research Studies in Biosciences (IJRSB). Volume 5, Issue 8, August 2017, PP 34-39.

[13] Martin, I. G., Marcias, E. M., Sanchez, J. S. and Rivera B. G. (1997). Detection of honey adulteration with beet sugar using stable isotope methodology. Food Chemistry, 61: 281-286.

[14] Mouazen, A. M. and Al-Walaan, N. (2014). Glucose Adulteration in Saudi Honey with Visible and Near Infrared Spectroscopy, International Journal of Food Properties, 17: 2263-2274.

[15] Lawal, R. A., A. K. Lawal, and J. B. Adekalu (2009). "Physico-chemical studies on adulteration of honey in Nigeria." Pakistan Journal of Biological Sciences 12.15: 1080.

[16] Ribeiro, R. D. O. R., Mársico, E. T., da Silva Carneiro, C., Monteiro, M. L. G., Júnior, C. C., \& de Jesus, E. F. O. (2014). Detection of honey adulteration of high fructose corn syrup by Low Field Nuclear Magnetic Resonance (LF 1H NMR). Journal of Food Engineering, 135, 39-43.

[17] Zhou, X., Taylor, M. P., Salouros H. and Prasad, S. (2018). Authenticity and geographic origin of global honeys determined using carbon isotope ratios and trace elements. Scientific reports, 8:14639. DOI: 10.1038/s41598-018-32764-w.

Citation: Meseret Gemeda, et.al, "Chemical and Physical Properties of Adulterated Honey and Developing Means of Identifying Adulterants", International Journal of Advanced Research in Chemical Science, 7(5), pp. 22-29. DOI: https:// doi.org/10.20431/2349-0403.0705003

Copyright: () 2020 Authors, this is an open-access article distributed under the terms of the Creative Commons Attribution License, which permits unrestricted use, distribution, and reproduction in any medium, provided the original author and source are credited. 